\title{
Postoperative Pulmonary Hemodynamics and Systemic Inflammatory Response in Pediatric Patients Undergoing Surgery for Congenital Heart Defects
}

\author{
Maria Francilene S. Souza $\left(\mathbb{D},{ }^{1}\right.$ Juliano G. Penha $\left(\mathbb{D},{ }^{1}\right.$ Nair Y. Maeda $\left(\mathbb{D},{ }^{2}\right.$ \\ Filomena R. B. G. Galas $\mathbb{D}^{1},{ }^{1}$ Kelly C. O. Abud $\mathbb{D},{ }^{1}$ Eloisa S. Carvalho $\mathbb{D}^{1}{ }^{1}$ \\ Ana Maria Thomaz $\mathbb{D}^{1},{ }^{1}$ Claudia R. P. Castro ${ }^{D},{ }^{1}$ Juliana Pereira $\left(\mathbb{D},{ }^{3}\right.$ \\ and Antonio Augusto Lopes $\mathbb{D}^{1}$ \\ ${ }^{1}$ Heart Institute (InCor), University of São Paulo School of Medicine, São Paulo, Brazil \\ ${ }^{2}$ Pró-Sangue Foundation, São Paulo, Brazil \\ ${ }^{3}$ Laboratory of Medical Investigation on Pathogenesis and Targeted Therapy in Onco-Immuno-Hematology (LIM-31), University of \\ São Paulo, São Paulo, Brazil \\ Correspondence should be addressed to Antonio Augusto Lopes; aablopes@usp.br
}

Received 30 September 2021; Revised 17 December 2021; Accepted 27 December 2021; Published 15 January 2022

Academic Editor: Fumio Tsuji

Copyright (c) 2022 Maria Francilene S. Souza et al. This is an open access article distributed under the Creative Commons Attribution License, which permits unrestricted use, distribution, and reproduction in any medium, provided the original work is properly cited.

\begin{abstract}
There is scarce information about the relationships between postoperative pulmonary hemodynamics, inflammation, and outcomes in pediatric patients with congenital cardiac communications undergoing surgery. We prospectively studied 40 patients aged $11(8-17)$ months (median with interquartile range) with a preoperative mean pulmonary arterial pressure of 48 (34-54) $\mathrm{mmHg}$ who were considered to be at risk for postoperative pulmonary hypertension. The immediate postoperative pulmonary/systemic mean arterial pressure ratio (PAP/SAP ${ }_{\text {IPO }}$, mean of first 4 values obtained in the intensive care unit, readings at 2-hour intervals) was correlated directly with PAP/SAP registered in the surgical room just after cardiopulmonary bypass $(r=0.68, p<0.001)$. For the entire cohort, circulating levels of 15 inflammatory markers changed after surgery. Compared with patients with PAP/SAP ${ }_{\mathrm{IPO}} \leq 0.40(n=22)$, those above this level $(n=18)$ had increased pre- and postoperative serum levels of granulocyte colony-stimulating factor $(p=0.040)$, interleukin-1 receptor antagonist $(p=0.020)$, interleukin- 6 $(p=0.003)$, and interleukin-21 ( $p=0.047)$ (panel for 36 human cytokines) and increased mean platelet volume $(p=0.018)$. Using logistic regression analysis, a PAP/SAP ${ }_{\mathrm{IPO}}>0.40$ and a heightened immediate postoperative serum level of macrophage migration inhibitory factor (quartile analysis) were shown to be predictive of significant postoperative cardiopulmonary events (respective hazard ratios with 95\% CIs, 5.07 (1.10-23.45), and 3.29 (1.38-7.88)). Thus, the early postoperative behavior of the pulmonary circulation and systemic inflammatory response are closely related and can be used to predict outcomes in this population.
\end{abstract}

\section{Introduction}

Pulmonary hypertension is an important factor influencing the outcomes following pediatric cardiac surgery. Young patients with unrestrictive congenital cardiac communications have altered pulmonary hemodynamics associated with functional and structural changes in the pulmonary vascular bed. These abnormalities pose a risk for immediate postoperative clinical instability and residual pulmonary hypertension long after the repair of cardiac lesions, particularly in individuals not treated in a timely fashion (early in life). A number of perioperative factors contribute to postoperative systemic and pulmonary vascular tone instability, leading to hemodynamic disturbances. Cardiopulmonary bypass $(\mathrm{CPB})$ with altered flow conditions, tissue ischemia and reperfusion, hypothermia, and the use of blood products 
or protamine elicit a systemic inflammatory response [1]. Superimposed on preexisting endothelial cell dysfunction, the inflammatory reaction elicits an increased propensity to spasm in the pulmonary circulation [2]. Cell activation (of endothelial cells, leukocytes, and platelets), which involves the expression of cytokines and adhesion molecules and the production and release of reactive oxygen species, arachidonic acid derivatives, and proteolytic enzymes are events that take place during and after $\mathrm{CPB}$, leading to vasomotor disturbances and multiorgan dysfunction [3-6]. Heightened circulating levels of endothelins and changes in the L-arginine-nitric oxide-cGMP pathway further contribute to pulmonary vascular reactivity [2]. The pulmonary vascular bed responds dramatically to changes in pH. Postoperatively, alveolar edema, ventilation-perfusion mismatch, and bronchoconstriction contribute to hypoxia, which may either trigger or exacerbate pulmonary vasoconstriction $[2,7]$.

In more privileged communities, the contemporary incidence of postoperative pulmonary hypertensive crises is probably less than $1 \%$ [2]. There have been estimates ranging between $2 \%$ and $5 \%$, with higher percentages in specific subsets of patients, such as those with Down syndrome $(\sim 10 \%)$ and those with atrioventricular septal defect (14\%) [8]. The lack of firm diagnostic criteria makes it difficult to have precise estimates of the incidence of crises. Very likely, the estimates of $\sim 1 \%$ refer to episodes of severe and sustained pulmonary artery pressure rise with hypoxemia and systemic hypotension, with still unacceptably high mortality rates of $20 \%$ to $30 \%$ [2, 9]. A pulmonary-to-systemic arterial pressure ratio above 0.75 with a decrease in systemic arterial pressure of $20 \%$ or more and a systemic oxygen saturation below $90 \%$ have been used as criteria to define pulmonary hypertensive crises [7]. In some reports, however, crises have been defined as a rise in pulmonary arterial pressure to or above the systemic arterial pressure level, with no further specifications about the duration of episodes or the occurrence of concomitant disturbances [10]. Strict definitions may be problematic in that they do not provide a realistic view of hemodynamic abnormalities that occur in this population. Transient elevations in pulmonary arterial pressure, even to the systemic level, that are promptly reversed by manual ventilation, sedation, and muscle relaxants probably do not have an impact on short-term outcomes. On the other hand, some episodes of pulmonary artery pressure rise, even if not severe enough to cause significant hemodynamic impairment, last longer, or occur in clusters, requiring substantial changes in therapeutic measures. In the presence of advanced right ventricular dysfunction, low cardiac output with systemic hypotension may be the dominant scenario rather than marked elevations of pulmonary arterial pressure [11]. While many of these instabilities are not necessarily associated with high mortality rates, they are major contributors to the need for prolonged mechanical ventilation and the length of stay in the hospital.

This study was planned to investigate the relationships between hemodynamics, postoperative clinical events, and inflammatory profiles in a prospective cohort of young pediatric patients undergoing surgery for congenital heart dis- ease who were considered to be at risk for postoperative pulmonary hypertension. This assumption of risk was based on the fact that they represented the population of a tertiary referral center with a high prevalence of Down syndrome and had unrestrictive cardiac communications with elevated pulmonary artery pressure, as suggested by preoperative echocardiographic evaluation. Postoperative hemodynamics was evaluated by continuously registering pulmonary and systemic arterial pressures for 2.5 days. We adopted a more flexible definition of postoperative cardiopulmonary events by including episodes that would not be interpreted as typical pulmonary hypertensive crises. However, we disregarded transient and isolated elevations in pulmonary arterial pressure. Inflammatory profiles were characterized by measuring the circulating levels of several inflammatory markers preoperatively and in the early postoperative period.

\section{Methods}

2.1. Study Population. This single-center study was performed at the Heart Institute (InCor), University of São Paulo School of Medicine, São Paulo, Brazil. Patients were consecutively enrolled from November 2016 to October 2018 based on the following criteria: age 1 month to 3 years; presence of unrestrictive cardiac communications, i.e., with a diameter of the posttricuspid communication greater than $50 \%$ of the aortic annulus diameter on transthoracic echocardiography; absence of pulmonary stenosis; hearts with biventricular physiology; clinical features suggestive of at least moderately elevated pulmonary arterial pressure; absence of extracardiac syndromes other than Down syndrome; and absence of any signs of ongoing or recent inflammatory or infectious diseases. In this study, patients were assigned to cardiac surgery essentially on the basis of noninvasive elevation. Patients with peripheral oxygen saturation lower than $85 \%$ and significant right-to-left shunting across the cardiac septal defects, suggesting the presence of advanced pulmonary vasculopathy, were not included. Healthy pediatric subjects with an age as close as possible to the age range of the patients were recruited from the same geographic area and entered the study as controls for the laboratory (biochemical) tests. This group was also made up of individuals with Down syndrome. Informed consent signed by family members was necessary for inclusion in all instances. The study protocol was approved by the Institutional Scientific and Ethics Committee (CEP n ${ }^{\circ}$. 2.068.696).

\subsection{Perioperative Protocols and Hemodynamic Parameters.} Some patients had clinical and radiographic signs of pulmonary overcirculation, often associated with a pulmonary/systemic blood flow ratio greater than 2.5 and peripheral oxygen saturation $>93 \%$. Subjects with no signs of congestive heart failure or failure to thrive, with a pulmonary/systemic blood flow ratio of $<2.0$ and peripheral oxygen saturation $\leq 93 \%$, were considered to have higher levels of pulmonary vascular resistance, so they required special attention perioperatively. Transthoracic echocardiography was performed preoperatively to assess anatomic and functional parameters. Systolic pulmonary artery pressure was 
obtained from the tricuspid regurgitation gradient. Mean pulmonary artery pressure was calculated as $(0.61 \times$ systolic pressure) $+2 \mathrm{mmHg}$ [12]. The pulmonary/systemic blood flow ratio and pulmonary venous flow were estimated based on flow parameters in the right and left ventricular outflow tracts and the velocity-time integral of blood flow in the pulmonary veins. Right ventricular systolic function was assessed by measuring the tricuspid annular plane systolic excursion (TAPSE).

Postoperatively, patients were kept under analgesia/sedation according to institutional protocols. In most instances, fentanyl, midazolam, and ketamine were used singly or in combination. Milrinone, epinephrine, and norepinephrine were used as inotropic/vasoactive agents. Inhaled nitric oxide (10-20 ppm) was started in the operating room immediately after CPB termination and was maintained throughout the entire period of mechanical ventilation. Pulmonary and systemic arterial pressures were measured directly in the operating room and by using indwelling catheters in the postoperative care unit. Intraoperatively, pressures were recorded before and after CPB. Postoperatively, readings were taken at 2-hour intervals (12 times a day) for 2.5 days. Pulmonary and systemic arterial pressures were computed, and pressure curves were constructed. The pulmonary/systemic mean arterial pressure ratio (PAP/SAP) was calculated. Immediate postoperative pulmonary hemodynamics was defined as the mean of the first 4 values (PAP/SAP $\left.{ }_{\text {IPO }}\right)$, corresponding to the first 6 hours of postoperative care. This parameter was used to characterize hemodynamic groups.

\subsection{Postoperative Cardiopulmonary Events (Study Outcome).} After surgery, we computed interrelated hemodynamic and respiratory disturbances of varying severity, from elevated pulmonary arterial pressure ( $>75 \%$ of systemic arterial pressure level) with a decline in systemic pressure $(\geq 20 \%)$ and oxygen desaturation $(<90 \%)$ to more severe hypoxemia and hypotension requiring manual ventilation and eventually cardiorespiratory resuscitation. In some instances, pulmonary arterial pressure was not seriously elevated at the onset of clinical instability (for example, $50 \%-75 \%$ of systemic pressure level). Rather, systemic hypotension was the dominant feature requiring changes in the doses of vasoactive-inotropic drugs. In the study, these disturbances are referred to as significant cardiopulmonary events (SCAPEs). Some episodes were prolonged (i.e., not promptly responsive to sedation and manual ventilation) and/or recurrent. Importantly, transient elevations of pulmonary pressure, even to suprasystemic levels, that were rapidly reversed by manual ventilation were not categorized as SCAPES. Interpretations were made independently by 3 physicians (AAL, AMT, and FRBGG). Patients whose systemic arterial pressure stabilized upon recovery from SCAPEs were started on sildenafil (four daily doses of $0.25 \mathrm{mg} / \mathrm{kg}$ via nasoenteral tube) and kept on it until nitric oxide discontinuation and weaning from mechanical ventilation or for the rest of their hospital stay.

\subsection{Microcirculatory Parameters and Inflammatory Markers.} Hemodynamics and microcirculatory status were monitored via pulmonary and systemic pressure curves, mixed venous oxygen saturation, and serum lactate, particularly on the first 2 to 3 days after surgery. The amount of cardiovascular support required postoperatively was computed using the vasoactive-inotropic score [13]. To investigate the relationships between the patterns of pressure curves, SCAPEs, and inflammatory profiles, inflammatory markers were analyzed preoperatively and 4 hours and 24 hours after CPB. We analyzed serum levels of 36 specific inflammatory mediators: complement component 5/5a (C5/C5a); CD40 ligand (CD40L); granulocyte colony-stimulating factor (G-CSF); granulocyte-macrophage colony-stimulating factor (GM$\mathrm{CSF})$; growth-regulated oncogene alpha (GRO $\alpha)$; human $\mathrm{CC}$ chemokine I-309 (I-309); intercellular adhesion molecule-1 (ICAM-1); interferon gamma (IFN- $\gamma$ ); interleukins (IL) 1 alpha, 1 beta, 2, 4, 5, 6, 8, 10, 12, 13, 16, 17, 17E, 18, 21, 27, and 32 alpha; interleukin-1 receptor antagonist (IL-1RA); interferon gamma-induced protein-10 (IP-10); interferoninducible $\mathrm{T}$ cell alpha chemoattractant (I-TAC); monocyte chemoattractant protein-1 (MCP-1); macrophage migration inhibitory factor (MIF); macrophage inflammatory protein-1 alpha/beta (MIP- $1 \alpha / \beta)$; regulated on activation, normal $\mathrm{T}$ cell expressed and secreted (RANTES); plasminogen activator inhibitor-1 (Serpin E1); stromal cell-derived factor-1 (SDF1); tumor necrosis factor alpha (TNF- $\alpha$ ); and soluble triggering receptor expressed on myeloid cell-1 (sTREM-1). Proteins were analyzed by immunoblotting using a human cytokine array (R\&D Systems, Minneapolis, MN, USA). Samples were processed in duplicate, and proteins were detected semiquantitatively by chemiluminescence. The results are expressed as the average signal of each pair of duplicate spots (units of pixel intensity, upi). For all patients, preoperative and postoperative samples were run in the same assay. Samples from pediatric controls were run in parallel. The control group was within the same age range of patients and included individuals with Down syndrome as well.

2.5. Data Obtainment. In this study, preoperative and postoperative assessments (responsible authors, MFSS, JGP, FRBGG, KCOA, ESC, AMT, and CRPC) and laboratory analyses (NYM) were carried out in a blinded fashion.

2.6. Statistical Analysis. Unless otherwise specified, numeric variables are presented as medians with interquartile ranges. Categorical variables are presented as number of cases and percentages, and differences between groups were tested using the Chi-square family of tests. The Wilcoxon test, Friedman's test, and Pearson's coefficient of correlation were used to test for differences and associations within subjects. Inferential statistics were used to analyze postoperative hemodynamics, biological markers, and microcirculatory parameters in hemodynamic groups, as well as potential predictors of outcome. For this purpose, the majority of variables were analyzed after Box-Cox transformation with further testing for the normality of their distributions. The general linear model was used to test for differences between groups at baseline and postoperatively. The general linear model for repeated measures was used to analyze arterial pressure and oxygen saturation curves postoperatively and differences between hemodynamic groups regarding data 
TABLe 1: Preoperative and intraoperative data of 40 patients.

\begin{tabular}{|c|c|}
\hline Age (months) & $11(8-17)$ \\
\hline $\operatorname{Sex}(M: F)$ & $11: 29$ \\
\hline Weight $(\mathrm{kg})$ & $6.42(5.72-7.91)$ \\
\hline Height $(\mathrm{cm})$ & $69(63-76)$ \\
\hline Down syndrome, $n(\%)$ & $25(62.5)$ \\
\hline Peripheral oxygen saturation (\%) & $96(93-98)$ \\
\hline \multicolumn{2}{|l|}{ Cardiac anomaly } \\
\hline Ventricular septal defect, $n(\%)^{*}$ & $24(60.0)$ \\
\hline Atrioventricular septal defect, $n(\%)^{\dagger}$ & $16(40.0)$ \\
\hline \multicolumn{2}{|l|}{ Echocardiographic parameters } \\
\hline Systolic pulmonary arterial pressure $(\mathrm{mmHg})$ & $75(52-86)$ \\
\hline Mean pulmonary arterial pressure $(\mathrm{mmHg})$ & $48(34-54)$ \\
\hline Pulmonary/systemic blood flow ratio & $2.30(1.80-3.20)$ \\
\hline Velocity-time integral of blood flow in pulmonary veins $(\mathrm{cm})^{\|}$ & $21.5(20.1-25.0)$ \\
\hline TAPSE $(\mathrm{mm})^{\ddagger}$ & $15.0(13.0-17.8)$ \\
\hline TAPSE, $Z$-score & $0.50(-1.60-1.93)$ \\
\hline \multicolumn{2}{|l|}{ Intraoperative parameters } \\
\hline Cardiopulmonary bypass (CPB) duration (min) & $128(90-152)$ \\
\hline \multicolumn{2}{|l|}{ Mean pulmonary arterial pressure } \\
\hline Before CPB (mmHg) & $32(26-36)$ \\
\hline After CPB (mmHg) & $22(18-26)^{\S}$ \\
\hline \multicolumn{2}{|l|}{ Mean systemic arterial pressure } \\
\hline Before CPB (mmHg) & $46(40-54)$ \\
\hline After CPB (mmHg) & $53(49-59)^{\S}$ \\
\hline \multicolumn{2}{|l|}{ Pulmonary/systemic mean arterial pressure ratio } \\
\hline Before CPB (mmHg) & $0.73(0.56-0.86)$ \\
\hline After CPB (mmHg) & $0.40(0.33-0.48)^{\S}$ \\
\hline Modified ultrafiltration volume $(\mathrm{mL})$ & $60.5(41.3-70.6)$ \\
\hline
\end{tabular}

Numeric variables are presented as median with interquartile range. ${ }^{*}$ Singly or in association with atrial septal defect and/or patent ductus arteriosus. ${ }^{\dagger}$ Singly or in association with patent ductus arteriosus. "Values $<20 \mathrm{~cm}$ are generally associated with heightened pulmonary vascular resistance in pediatric patients with unrestrictive cardiac communications $[14,15] .{ }^{*}$ Tricuspid annular plane systolic excursion. Normally, values increase with increasing age in the pediatric population. A TAPSE of $\geq 15.5$ is considered normal at the age of 1 year, as are values $\geq 16.5$ by the age of 2 years [16]. ${ }^{\$} p<0.001$ versus pre-CPB levels, Wilcoxon test.

obtained at baseline and 4 hours and 24 hours after surgery. Logistic regression (univariate and multivariate analyses) was used to identify possible predictors of postoperative cardiopulmonary events. Receiver operating characteristic (ROC) curves were constructed, and sensitivity and specificity levels associated with cutoff values were determined. In all tests, 0.05 was set as the significance level. Statistical analysis was performed using the SPSS statistical software, version 26 (IBM, Armonk, NY, USA).

\section{Results}

3.1. Descriptive Analysis. Preoperative and intraoperative data of 40 patients who underwent surgery under CPB are listed in Table 1. There was a high proportion of patients with Down syndrome presenting with a complete atrioventricular septal defect. Elevation of pulmonary vascular resistance was suggested by increased pulmonary arterial pressure in the presence of relatively restricted pulmonary blood flow considering the size of the cardiac communications. Preoperative right ventricular systolic function (TAPSE) was roughly normal in most subjects. Pulmonary hypertension was further confirmed by an intraoperative pulmonary/systemic mean arterial pressure ratio of 0.73 (0.56-0.86) (median with interquartile range). Pulmonary arterial pressure decreased significantly after $\mathrm{CPB}$ in comparison with pre-CPB level, but it remained at $\sim 40 \%$ of the systemic arterial pressure level (Table 1). Despite the use of inhaled nitric oxide, which was started in the operating room and maintained throughout the period of mechanical ventilation, 11 patients had SCAPEs of varying severity postoperatively. SCAPEs contributed to prolonged mechanical ventilation $(p<0.001)$ but not mortality. Other factors associated with prolonged mechanical ventilation ( $>7$ days in 22 patients) were delayed sternal closure, respiratory disturbances, infection, arrhythmias, seizures, and other neurological manifestations. One patient died of septicemia. Another patient died of rapid-onset systemic hypotension followed 


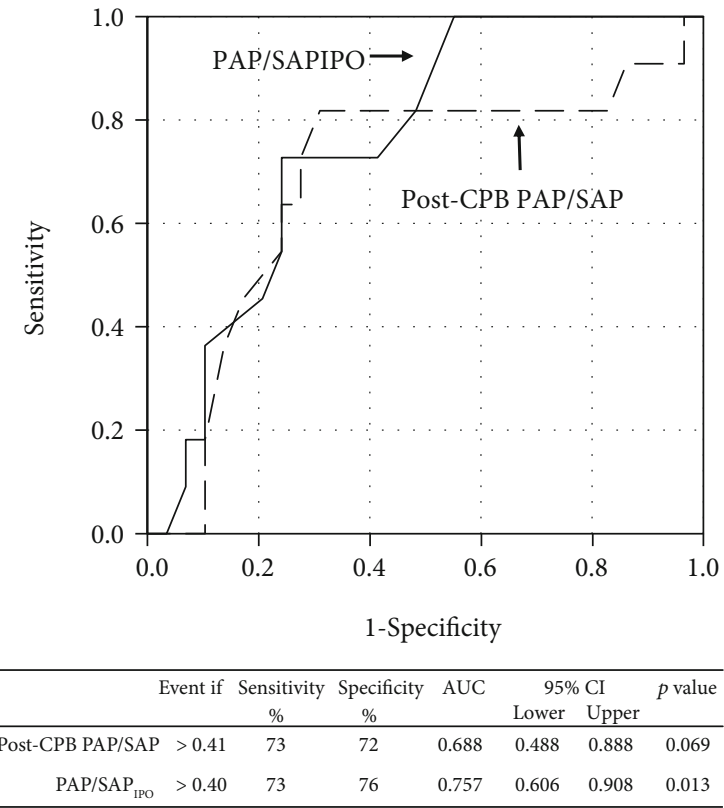

FIGURE 1: Receiver operating characteristic curves and cutoff values for pulmonary/systemic mean arterial pressure ratio (PAP/SAP) obtained just after cardiopulmonary bypass (post-CPB) and during the immediate postoperative period (IPO) in the prediction of significant cardiopulmonary events during the intensive care unit stay.

by bradycardia unresponsive to vasopressin and other lifesupporting interventions. There was no need for reoperation in the present cohort.

3.2. Hemodynamic Groups. While analyzing postoperative hemodynamics, we observed that PAP/SAP ${ }_{\text {IPO }}(0.38(0.32-$ $0.46)$, range 0.19 to 0.67 ) was directly correlated with the $\mathrm{PAP} / \mathrm{SAP}$ registered in the surgical room just after $\mathrm{CPB}$ discontinuation $(r=0.68, p<0.001)$. PAP/SAP ${ }_{\text {IPO }}$ was even better than post-CPB PAP/SAP at predicting SCAPEs (Figure 1). The cutoff value of 0.40 obtained for PAP/SAP was very close to the median value of 0.38 . Based on these observations, we decided to use $\mathrm{PAP} / \mathrm{SAP}_{\mathrm{IPO}}(\leq 0.40$ versus $>0.40$ ) to categorize postoperative pulmonary hemodynamics and analyze factors that might explain differences in hemodynamic behavior. Compared with patients with PAP $/ \mathrm{SAP}_{\mathrm{IPO}} \leq 0.40 \quad(n=22)$, those with $\mathrm{PAP} / \mathrm{SAP}_{\mathrm{IPO}}>0.40$ $(n=18)$ had greater pulmonary arterial pressure for the entire period of hemodynamic monitoring (Figure 2). Systemic arterial pressure did not differ between the groups, while peripheral oxygen saturation tended (nonsignificantly) to be lower in subjects with $\mathrm{PAP} / \mathrm{SAP}_{\mathrm{IPO}}>0.40$ during the first 36 hours. Preoperative and intraoperative data in hemodynamic groups are shown in Table 2. In the group of patients with $\mathrm{PAP} / \mathrm{SAP}_{\mathrm{IPO}}>0.40$, there was a greater proportion of individuals with an atrioventricular septal defect. A longer time was required to repair their cardiac lesions (CPB duration) in comparison with patients with PAP/SA $\mathrm{P}_{\mathrm{IPO}} \leq 0.40$. Besides, in patients with $\mathrm{PAP} / \mathrm{SAP}_{\mathrm{IPO}}>0.40$, preoperative oxygen saturation was slightly but significantly lower. Notably, atrioventricular septal defects were present
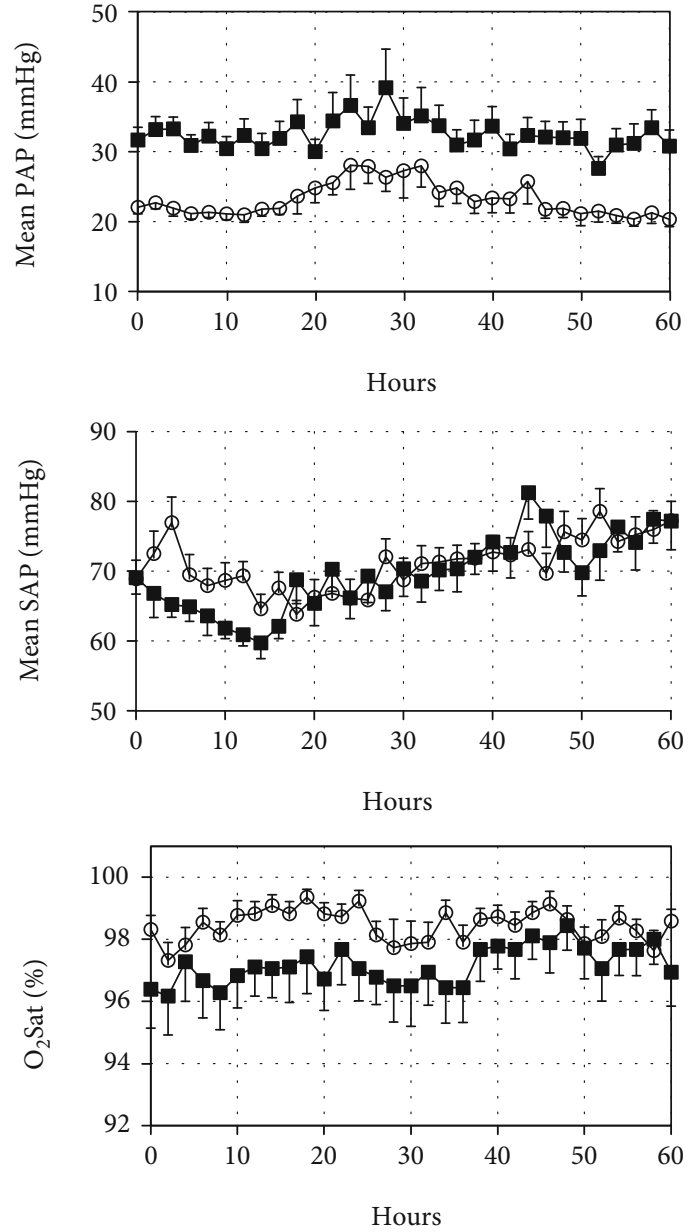

FIgURe 2: Pulmonary arterial pressure (PAP), systemic arterial pressure (SAP), and peripheral oxygen saturation $\left(\mathrm{O}_{2}\right.$ Sat) measured in the postoperative care unit with readings at 2-hour intervals (12 times within a day) for 2.5 consecutive days. Data are presented as mean with SE. Circles and squares represent patients with $\mathrm{PAP} / \mathrm{SAP} \mathrm{IPO}_{\mathrm{IPO}}$ (immediate postoperative period) $\leq 0.40$ $(n=22)$ and $>0.40 \quad(n=18)$, respectively. Data were analyzed using the general linear model for repeated measures. There was a significant between-group difference for mean PAP $(p<0.001)$ but not for mean SAP $(p=0.439)$ or $\mathrm{O}_{2}$ Sat $(0.113)$.

exclusively in subjects with Down syndrome. CPB time was 144 (108-157) minutes and 103 (90-125) minutes in syndromic and nonsyndromic individuals, respectively $(p=0.009)$. Also, Down syndrome patients had lower preoperative peripheral oxygen saturation compared with nonsyndromic subjects (96\% (93\%-98\%) and 98\% (96\%-99\%), respectively, $p=0.006)$.

3.3. Hemodynamics and Inflammatory Response. Preoperative and postoperative hematological parameters are shown in Table 3. Postoperatively, there was a decrease in lymphocyte count, while circulating monocytes and neutrophils and the neutrophil-to-lymphocyte ratio increased, compared to baseline. There was a decline in platelet count (no platelet transfusions we required) while the mean platelet volume and plasma C-reactive protein increased. The preoperative 
TABLE 2: Preoperative and intraoperative data in hemodynamic groups.

\begin{tabular}{|c|c|c|c|}
\hline & $\mathrm{PAP} / \mathrm{SAP}_{\mathrm{IPO}} \leq 0.40(n=22)$ & $\mathrm{PAP} / \mathrm{SAP}_{\mathrm{IPO}}>0.40(n=18)$ & $p$ value \\
\hline Age (months) & $12(8-20)$ & $11(8-15)$ & 0.874 \\
\hline $\operatorname{Sex}(M: F)$ & $6: 16$ & $5: 13$ & $0.999^{*}$ \\
\hline Down syndrome, $n(\%)$ & $11(50)$ & $14(78)$ & $0.140^{\dagger}$ \\
\hline Peripheral oxygen saturation (\%) & $98(96-99)$ & $95(92-96)$ & $<0.001$ \\
\hline Atrioventricular septal defect: ventricular septal defect & $5: 17$ & $11: 7$ & $0.032^{\dagger}$ \\
\hline Pulmonary/systemic blood flow ratio & $2.45(1.80-3.33)$ & $2.15(1.68-2.98)$ & 0.360 \\
\hline $\begin{array}{l}\text { Precardiopulmonary bypass }(\mathrm{CPB}) \\
\text { mean pulmonary arterial pressure }(\mathrm{mmHg})\end{array}$ & $31(23-35)$ & $35(29-39)$ & 0.146 \\
\hline Pre-CPB pulmonary/systemic mean arterial pressure ratio & $0.65(0.50-0.80)$ & $0.77(0.66-0.87)$ & 0.091 \\
\hline $\mathrm{CPB}$ duration (min.) & $107(86-135)$ & $146(121-159)$ & 0.007 \\
\hline
\end{tabular}

Numeric variables are presented as median with interquartile range. Differences were analyzed using the general linear model after Box-Cox transformation of dependent variables. ${ }^{*}$ Fisher's exact test. ${ }^{\dagger}$ Chi-square test.

TABle 3: Preoperative and postoperative hematological parameters.

\begin{tabular}{|c|c|c|c|c|c|}
\hline & \multirow{2}{*}{ Before surgery } & \multicolumn{3}{|c|}{ Post-CPB } & \multirow{2}{*}{$p$ value $^{*}$} \\
\hline & & $4 \mathrm{~h}$ & $24 \mathrm{~h}$ & $48 \mathrm{~h}$ & \\
\hline White blood cell count $(\mathrm{K} / \mu \mathrm{L})$ & $\begin{array}{c}11.04 \\
(7.14-12.21)\end{array}$ & $\begin{array}{c}14.06 \\
(11.02-17.66)^{\dagger}\end{array}$ & $\begin{array}{c}14.17 \\
(11.86-18.09)^{\dagger}\end{array}$ & $\begin{array}{c}14.75 \\
(12.13-20.19)^{\dagger}\end{array}$ & $<0.001$ \\
\hline Lymphocytes $(\mathrm{K} / \mu \mathrm{L})$ & $\begin{array}{c}4.66 \\
(3.66-7.76)\end{array}$ & $\begin{array}{c}1.70 \\
(1.02-2.41)^{\dagger}\end{array}$ & $\begin{array}{c}1.87 \\
(1.22-2.23)^{\dagger}\end{array}$ & $\begin{array}{c}2.37 \\
(1.51-2.98)^{\dagger}\end{array}$ & $<0.001$ \\
\hline Neutrophils $(\mathrm{K} / \mu \mathrm{L})$ & $\begin{array}{c}3.33 \\
(2.00-4.91)\end{array}$ & $\begin{array}{c}10.59 \\
(8.28-13.86)^{\dagger}\end{array}$ & $\begin{array}{c}10.64 \\
(8.35-13.66)^{\dagger}\end{array}$ & $\begin{array}{c}10.97 \\
(7.61-15.29)^{\dagger}\end{array}$ & $<0.001$ \\
\hline Neutrophil to lymphocyte ratio & $\begin{array}{c}0.57 \\
(0.35-1.05)\end{array}$ & $\begin{array}{c}6.83 \\
(4.06-10.88)^{\dagger}\end{array}$ & $\begin{array}{c}5.92 \\
(4.28-9.25)^{\dagger}\end{array}$ & $\begin{array}{c}5.07 \\
(2.94-9.13)^{\dagger}\end{array}$ & $<0.001$ \\
\hline Monocytes $(\mathrm{K} / \mu \mathrm{L})$ & $\begin{array}{c}0.71 \\
(0.50-1.04)\end{array}$ & $\begin{array}{c}1.03 \\
(0.73-1.48)^{\dagger}\end{array}$ & $\begin{array}{c}1.47 \\
(0.86-1.93)^{\dagger}\end{array}$ & $\begin{array}{c}1.34 \\
(0.85-1.81)^{\dagger}\end{array}$ & $<0.001$ \\
\hline Platelets $(\mathrm{K} / \mu \mathrm{L})$ & $\begin{array}{c}329 \\
(272-390)\end{array}$ & $\begin{array}{c}141 \\
(118-170)^{\dagger}\end{array}$ & $\begin{array}{c}136 \\
(110-164)^{\dagger}\end{array}$ & $\begin{array}{c}138 \\
(107-178)^{\dagger}\end{array}$ & $<0.001$ \\
\hline Mean platelet volume (fL) & $\begin{array}{c}9.50 \\
(9.00-10.00)\end{array}$ & $\begin{array}{c}9.40 \\
(8.80-9.90)\end{array}$ & $\begin{array}{c}10.05 \\
(9.45-10.40)^{\ddagger}\end{array}$ & $\begin{array}{c}10.30 \\
(9.75-10.70)^{\ddagger}\end{array}$ & $<0.001$ \\
\hline C-reactive protein $(\mathrm{mg} / \mathrm{L})$ & $\begin{array}{c}2.07 \\
(1.17-4.57)\end{array}$ & $\begin{array}{c}2.88 \\
(1.78-3.76)\end{array}$ & $\begin{array}{c}69.50 \\
(53.80-81.80)^{\dagger}\end{array}$ & $\begin{array}{c}136.19 \\
(108.24-201.06)^{\dagger}\end{array}$ & $<0.001$ \\
\hline
\end{tabular}

Variables are presented as median with interquartile range. ${ }^{*}$ Friedman test. $\dagger$ and $\neq$, respectively, $p<0.001$ and $p<0.01$ versus baseline $(p o s t$ hoc multiple comparisons). CPB: cardiopulmonary bypass.

levels of the specific inflammatory proteins investigated in this study did not differ significantly between patients and controls, except for the chemokine RANTES, which was higher in patients (Table 4). Inflammatory markers were further analyzed for possible differences within subjects and between hemodynamic groups as defined. The levels of several markers changed significantly within subjects (measurements performed before surgery versus 4 hours and 24 hours after CPB termination) in both groups (Table 5). For example, IL-1RA was markedly elevated 4 hours after CPB compared to baseline, and it later declined. Similar increases were observed for other proteins, whereas the levels of CD40L, GRO $\alpha$, Serpin E1, RANTES, and SDF-1 decreased after surgery. Serum CD40L correlated positively with platelet count before surgery $(r=0.41, p=0.012)$ and 4 hours and 24 hours following CPB $(r=0.35, p=0.035$ and $r=0.41$, $p=0.011$, respectively). In addition, there was a negative correlation between serum RANTES and vasoactive-inotropic score $(r=-0.57, p<0.001$ and $r=-0.32, p=0.043$, respectively, 4 hours and 24 hours after CPB). Differences between the 2 groups were observed in 5 markers, namely, G-CSF, IL1RA, IL-6, IL-21, and mean platelet volume, all of them higher in patients with PAP/SAP ${ }_{\text {IPO }}>0.40$ (Table 5).

3.4. Outcome Prediction. By univariate and multivariate logistic regression analyses, variables were tested for their association with SCAPEs. Table 6 shows the variables for which a $p$ value of $<0.10$ was obtained in univariate analysis. Except for the neutrophil-to-lymphocyte ratio, which appeared in the regression analysis with a negative coefficient, the variables shown in the table were positively correlated with the occurrence of SCAPEs. Early postoperative 
TABLE 4: Baseline levels of inflammatory proteins.

\begin{tabular}{|c|c|c|c|c|c|c|c|}
\hline & Controls $(n=37)$ & Patients $(n=40)$ & $p$ value & & Controls $(n=37)$ & Patients $(n=40)$ & $p$ value \\
\hline C5/C5a (upi) & $\begin{array}{c}1392 \\
(804-3756)\end{array}$ & $\begin{array}{c}2442 \\
(1027-3980)\end{array}$ & 0.209 & IL-13 (upi) & $\begin{array}{c}509 \\
(294-1090)\end{array}$ & $\begin{array}{c}654 \\
(311-1256)\end{array}$ & 0.492 \\
\hline CD40L (upi) & $\begin{array}{c}4353 \\
(2670-7079)\end{array}$ & $\begin{array}{c}5233 \\
(2871-7770)\end{array}$ & 0.677 & IL-16 (upi) & $\begin{array}{c}521 \\
(276-1116)\end{array}$ & $\begin{array}{c}568 \\
(262-1240)\end{array}$ & 0.749 \\
\hline G-CSF (upi) & $\begin{array}{c}347 \\
(198-603)\end{array}$ & $\begin{array}{c}560 \\
(245-867)\end{array}$ & 0.120 & IL-17 (upi) & $\begin{array}{c}257 \\
(146-450)\end{array}$ & $\begin{array}{c}300 \\
(190-556)\end{array}$ & 0.204 \\
\hline GM-CSF (upi) & $\begin{array}{c}293 \\
(146-356)\end{array}$ & $\begin{array}{c}343 \\
(168-492)\end{array}$ & 0.450 & IL-17E (upi) & $\begin{array}{c}344 \\
(183-565)\end{array}$ & $\begin{array}{c}407 \\
(224-786)\end{array}$ & 0.173 \\
\hline GRO $\alpha$ (upi) & $\begin{array}{c}1712 \\
(1315-2578)\end{array}$ & $\begin{array}{c}1770 \\
(1204-2866)\end{array}$ & 0.824 & IL-27 (upi) & $\begin{array}{c}221 \\
(134-411)\end{array}$ & $\begin{array}{c}304 \\
(173-422)\end{array}$ & 0.401 \\
\hline I-309 (upi) & $\begin{array}{c}636 \\
(318-802)\end{array}$ & $\begin{array}{c}674 \\
(255-1210)\end{array}$ & 0.301 & IL-32 $\alpha$ (upi) & $\begin{array}{c}470 \\
(220-980)\end{array}$ & $\begin{array}{c}447 \\
(257-990)\end{array}$ & 0.993 \\
\hline ICAM-1 (upi) & $\begin{array}{c}38777 \\
(30494-49330)\end{array}$ & $\begin{array}{c}39756 \\
(29716-48504)\end{array}$ & 0.847 & IP-10 (upi) & $\begin{array}{c}646 \\
(246-1185)\end{array}$ & $\begin{array}{c}827 \\
(432-1941)\end{array}$ & 0.052 \\
\hline IFN- $\gamma$ (upi) & $\begin{array}{c}269 \\
(168-389)\end{array}$ & $\begin{array}{c}329 \\
(190-438)\end{array}$ & 0.434 & I-TAC (upi) & $\begin{array}{c}522 \\
(243-989)\end{array}$ & $\begin{array}{c}706 \\
(309-1024)\end{array}$ & 0.459 \\
\hline IL-1 $\alpha$ (upi) & $\begin{array}{c}363 \\
(169-589)\end{array}$ & $\begin{array}{c}423 \\
(208-712)\end{array}$ & 0.407 & MCP-1 (upi) & $\begin{array}{c}345 \\
(261-637)\end{array}$ & $\begin{array}{c}491 \\
(238-876)\end{array}$ & 0.319 \\
\hline IL- $1 \beta$ (upi) & $\begin{array}{c}191 \\
(100-386)\end{array}$ & $\begin{array}{c}185 \\
(116-515)\end{array}$ & 0.766 & MIF (upi) & $\begin{array}{c}6339 \\
(5432-6838)\end{array}$ & $\begin{array}{c}5677 \\
(4635-7169)\end{array}$ & 0.444 \\
\hline IL-1RA (upi) & $\begin{array}{c}1407 \\
(759-2276)\end{array}$ & $\begin{array}{c}1571 \\
(605-2493)\end{array}$ & 0.903 & MIP- $1 \alpha / \beta$ (upi) & $\begin{array}{c}409 \\
(232-580)\end{array}$ & $\begin{array}{c}601 \\
(249-914)\end{array}$ & 0.093 \\
\hline IL-2 (upi) & $\begin{array}{c}268 \\
(119-415)\end{array}$ & $\begin{array}{c}324 \\
(121-511)\end{array}$ & 0.378 & Serpin E1 (upi) & $\begin{array}{c}44804 \\
(40018-51686)\end{array}$ & $\begin{array}{c}46656 \\
(35898-61188)\end{array}$ & 0.670 \\
\hline IL-4 (upi) & $\begin{array}{c}276 \\
(179-444)\end{array}$ & $\begin{array}{c}344 \\
(208-510)\end{array}$ & 0.333 & RANTES (upi) & $\begin{array}{c}58061 \\
(47531-65624)\end{array}$ & $\begin{array}{c}66140 \\
(53982-76405)\end{array}$ & 0.012 \\
\hline IL-5 (upi) & $\begin{array}{c}98 \\
(56-198)\end{array}$ & $\begin{array}{c}94 \\
(76-226)\end{array}$ & 0.601 & SDF-1 (upi) & $\begin{array}{c}3892 \\
(3250-5036)\end{array}$ & $\begin{array}{c}4251 \\
(3323-5967)\end{array}$ & 0.419 \\
\hline IL-6 (upi) & $\begin{array}{c}560 \\
(260-983)\end{array}$ & $\begin{array}{c}301 \\
(192-718)\end{array}$ & 0.197 & TNF- $\alpha$ (upi) & $\begin{array}{c}260 \\
(144-473)\end{array}$ & $\begin{array}{c}342 \\
(151-629)\end{array}$ & 0.508 \\
\hline IL-8 (upi) & $\begin{array}{c}202 \\
(138-396)\end{array}$ & $\begin{array}{c}282 \\
(155-509)\end{array}$ & 0.272 & sTREM-1 (upi) & $\begin{array}{c}264 \\
(151-543)\end{array}$ & $\begin{array}{c}381 \\
(235-595)\end{array}$ & 0.312 \\
\hline IL-10 (upi) & $\begin{array}{c}276 \\
(132-399)\end{array}$ & $\begin{array}{c}277 \\
(197-490)\end{array}$ & 0.809 & IL-18 (upi) & $\begin{array}{c}874 \\
(500-1718)\end{array}$ & $\begin{array}{c}951 \\
(527-1602)\end{array}$ & 0.909 \\
\hline IL-12p70 (upi) & $\begin{array}{c}243 \\
(119-465)\end{array}$ & $\begin{array}{c}261 \\
(142-443)\end{array}$ & 0.612 & IL-21 (upi) & $\begin{array}{c}401 \\
(185-612)\end{array}$ & $\begin{array}{c}379 \\
(253-789)\end{array}$ & 0.512 \\
\hline
\end{tabular}

Data are presented as median with interquartile range. Levels of inflammatory markers are given in units of pixel intensity (upi, chemiluminescence). Differences were analyzed using the general linear model after Box-Cox transformation of dependent variables.

hemodynamics (PAP/SAP $\left.{ }_{\mathrm{IPO}}\right)$ and IL-16, MIF, and neutrophil-to-lymphocyte ratio measured 4 hours after $\mathrm{CPB}$ were associated with a $p<0.05$. However, using the forward LR procedure for variable inclusion, only PAP/SAP IPO and MIF remained in the predictive model. The hazard ratio associated with a PAP/SAP ${ }_{\text {IPO }}>0.40$ was 5.07 (95\% CI $1.10-$ $23.45, p=0.038)$; for post-CPB MIF analyzed as quartiles, it was 3.29 (95\% CI $1.38-7.88, p<0.001)$. Variables not significantly correlated with the occurrence of SCAPEs were patient age and presence/absence of Down syndrome; preoperative oxygen saturation, pulmonary/systemic blood flow ratio, and right ventricular systolic function (TAPSE); preand post-CPB pulmonary/systemic mean arterial pressure ratio and $\mathrm{CPB}$ time; postoperative vasoactive inotropic score, serum lactate, and mixed venous oxygen saturation; and serum levels of inflammatory markers (pre- and postoperative) not shown in Table 6.

\section{Discussion}

In this study, we analyzed the behavior of pulmonary and systemic arterial pressure curves and the occurrence of SCAPEs and investigated how they might be related to systemic inflammation, which we assessed by measuring circulating levels of inflammatory markers pre- and postoperatively. Pulmonary hemodynamic patterns were identified early after surgery and correlated with the levels of 5 inflammatory markers. SCAPEs were related to immediate postoperative hemodynamics and serum level of MIF protein and constituted one of the causes of prolonged mechanical 


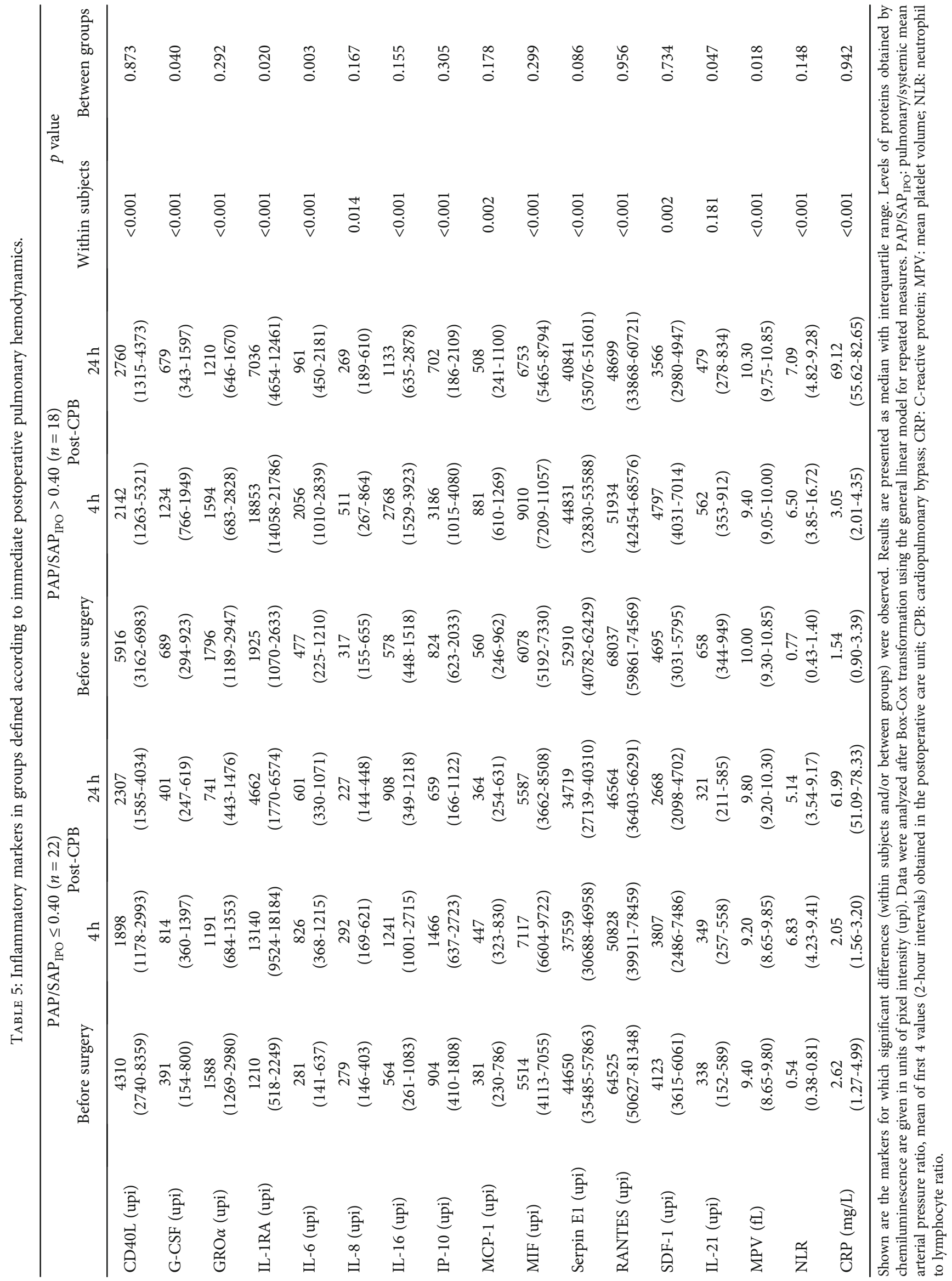


TABLE 6: Variables associated with significant postoperative cardiopulmonary events (SCAPEs).

\begin{tabular}{|c|c|c|c|c|c|}
\hline & \multicolumn{2}{|c|}{ SCAPEs } & \multicolumn{3}{|c|}{$p$ value } \\
\hline & No $(n=29)$ & Yes $(n=11)$ & Univariate analysis & Multivariate analysis 1 & Multivariate analysis 2 \\
\hline $\mathrm{PAP} / \mathrm{SAP}_{\mathrm{IPO}}>0.40, n(\%)$ & $\begin{array}{c}10 \\
(35)\end{array}$ & $\begin{array}{c}8 \\
(73)\end{array}$ & 0.038 & 0.046 & 0.047 \\
\hline CD40L at baseline (upi) & $\begin{array}{c}4348 \\
(2871-6863)\end{array}$ & $\begin{array}{c}6163 \\
(3642-11641)\end{array}$ & 0.078 & 0.291 & - \\
\hline IL-6 at baseline (upi) & $\begin{array}{c}281 \\
(180-637)\end{array}$ & $\begin{array}{c}499 \\
(179-1267)\end{array}$ & 0.079 & 0.406 & - \\
\hline IL- 8 at baseline (upi) & $\begin{array}{c}188 \\
(119-351)\end{array}$ & $\begin{array}{c}403 \\
(283-618)\end{array}$ & 0.089 & 0.767 & - \\
\hline IL-16 on day 0 (upi) & $\begin{array}{c}1241 \\
(956-2767)\end{array}$ & $\begin{array}{c}2140 \\
(1265-5703)\end{array}$ & 0.048 & 0.859 & - \\
\hline IL-1RA on day 0 (upi) & $\begin{array}{c}14181 \\
(9916-19524)\end{array}$ & $\begin{array}{c}20302 \\
(14144-22796)\end{array}$ & 0.078 & 0.446 & - \\
\hline MIF on day 0 (upi) & $\begin{array}{c}7221 \\
(6660-9356)\end{array}$ & $\begin{array}{c}10851 \\
(7224-14947)\end{array}$ & 0.021 & 0.059 & 0.023 \\
\hline RANTES on day 0 (upi) & $\begin{array}{c}49528 \\
(41541-59176)\end{array}$ & $\begin{array}{c}69437 \\
(40039-95821)\end{array}$ & 0.080 & 0.451 & - \\
\hline SDF-1 on day 0 (upi) & $\begin{array}{c}4131 \\
(2477-6277)\end{array}$ & $\begin{array}{c}6584 \\
(3931-10135)\end{array}$ & 0.075 & 0.878 & - \\
\hline NLR on day 0 & $\begin{array}{c}7.58 \\
(4.42-11.71)\end{array}$ & $\begin{array}{c}3.79 \\
(2.24-6.82)\end{array}$ & 0.043 & 0.105 & - \\
\hline $\mathrm{CRP}$ on day $2(\mathrm{mg} / \mathrm{L})$ & $\begin{array}{c}118.89 \\
(95.70-193.51)\end{array}$ & $\begin{array}{c}163.43 \\
(136.59-213.60)\end{array}$ & 0.077 & 0.419 & - \\
\hline
\end{tabular}

Variables were tested using logistic regression. Shown are those with $p<0.10$ in univariate analysis. In multivariate analysis 1 , all variables were included in the

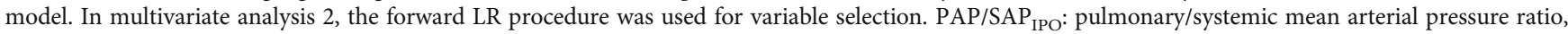
mean of first 4 values obtained at the postoperative care unit; CRP: C-reactive protein; NLR: neutrophil to lymphocyte ratio; baseline: day 0 and day 2 , respectively, preoperative determination, 4 hours after cardiopulmonary bypass and 2 days after surgery.

ventilation. The variable $\mathrm{PAP} / \mathrm{SAP}_{\mathrm{IPO}}$ was characterized as a new and potentially useful parameter. We looked at PAP/ $\mathrm{SAP}_{\text {IPO }}$ prior to analyzing the entire pressure curves because during the first 6 hours of postoperative care, patients were still deeply sedated on mechanical ventilation and relatively stabilized in terms of post-CPB physiology. Our findings suggest that the link between Down syndrome and pulmonary hypertension in the setting of congenital heart disease is constituted by features that are present in some but not all syndromic patients (e.g., atrioventricular septal defect and systemic oxygen desaturation) and becomes more evident under certain circumstances (longer surgical times). In some patients with Down syndrome, particularly those with atrioventricular septal defect, long perfusion times probably contributed to the upward shift of pulmonary pressure curve after surgery, which was clearly associated with systemic inflammation. Patients with this profile seemed to be at a higher risk of having a difficult postoperative course.

The overall postoperative inflammatory reaction was characterized by changes in the levels of several inflammatory markers, as shown in Table 4 . The levels of most markers increased from baseline, while the levels of 5 proteins actually decreased after surgery: CD40L, RANTES, GRO $\alpha$, Serpin E1, and SDF-1. We were probably unable to detect higher levels for GRO $\alpha$, Serpin E1, and SDF-1 because peak concentrations of these proteins generally occur just after or even during CPB [17-19]. The decrease in CD40L was probably a result of the decrease in the number of circulating platelets after surgery. Platelets are a major source of CD40L [20] in addition to its expression by activated CD4 $+\mathrm{T}$ cells [21]. In our patients, serum levels of CD40L correlated positively with platelet counts pre- and postoperatively. The chemokine RANTES was increased in patients versus controls preoperatively, confirming previous observations [22]. RANTES declined postoperatively, probably as a result of the use of inotropic and vasopressor agents. RANTES is produced by human vascular endothelial cells under proinflammatory conditions [23], as well as being expressed by activated T cells and platelets [24]. In pediatric cardiac surgery, circulating RANTES has been shown to decrease, with lower levels being associated with longer surgical times, inotropic support, and reoperation [25]. In our study, postoperative RANTES levels correlated negatively with the vasoactive-inotropic score. Furthermore, CPB can cause a marked reduction in T lymphocytes [26] and the concentrations of macrophage-recruiting chemokines, including MIP$1 \beta$ and RANTES. The recruitment and activation of neutrophil granulocytes are associated with multiorgan injury [27]. In the present study, CPB was followed by a decrease in lymphocyte count with a marked rise in the neutrophil-tolymphocyte ratio.

Of the inflammatory markers that were analyzed in the study, IL-6, IL-1RA, G-CSF, IL-21, and mean platelet volume were closely correlated with early postoperative 
hemodynamic patterns, while higher MIF concentrations 4 hours post-CPB were associated with the occurrence of SCAPEs. In general, IL-6 and IL-1RA are proinflammatory and anti-inflammatory mediators, respectively. G-CSF and IL-21 have effects that characterize them as antiinflammatory cytokines $[28,29]$. In the pathophysiological context of this study, the increase in platelet volume may be interpreted as a proinflammatory event [30]. In pediatric cardiac surgery, increased plasma and tissue concentrations of proinflammatory cytokines such as IL- 6 and IL- 8 are balanced by a phased plasma (IL-10 followed by IL-1RA) and tissue (IL-10) anti-inflammatory responses [31, 32]. The $\sim 10$-fold increase in serum IL-1RA that we observed postoperatively, in the absence of any significant increase in the level of IL- $1 \alpha$ or IL-1 $\beta$ (not shown), was not surprising. Because of the spare receptor effect, large quantities $(\sim 100$ fold) of IL-1RA are required to functionally inhibit the biological effects of negligible amounts of IL-1 [33]. Thus, in the study, the increase in IL-1RA concentration actually reflects increased postoperative IL-1 activity. IL-1RA, IL-10, and MCP-1 were shown to play an important role in the recovery of monocyte function after pediatric cardiac surgery [34]. There have been studies on the pathophysiological role of IL-1RA in experimental pulmonary hypertension [35-37], but there are no studies in pediatric pulmonary hypertension, nor are there studies in the particular setting of perioperative pulmonary hypertension. Our data seem to indicate that both proinflammatory (IL-6) and antiinflammatory responses (IL-1RA, G-CSF, and IL-21) were more prominent in patients with heightened postoperative pulmonary artery pressure. We speculate that the antiinflammatory response, albeit evident, was not sufficiently intense to antagonize the effects of proinflammatory stimuli on pulmonary vascular tone in these individuals.

SCAPEs occurred in $27.5 \%$ of patients. This relatively high prevalence was due to the inclusion of events that would probably not be classified as pulmonary hypertensive crises based on contemporary definitions but contributed to the need for prolonged mechanical ventilation. Of the variables tested for correlation with the occurrence of SCAPEs, immediate postoperative hemodynamics and serum MIF 4 hours post-CPB reached statistical significance in univariate and multivariate analyses. Higher initial levels of pulmonary arterial pressure (i.e., PAP/SAP ${ }_{\mathrm{IPO}}>0.40$ ) may have rendered some patients more susceptible to instabilities triggered by factors known to affect pulmonary vascular tone postoperatively, such as alveolar hypoxia, changes in $\mathrm{pH}$, and right ventricular dysfunction. MIF is a noncanonical ligand of chemokine receptors known to play a central role in innate immunity [38] and a number of biological processes involved in vascular remodeling, including pulmonary vascular remodeling [39]. It binds to the receptors CXCR2, CXCR4, and CXCR2/CD74, leading to the activation of signaling pathways that results in vascular smooth muscle cell proliferation and inhibition of endothelial cell apoptosis, recruitment of peripheral blood mononuclear cells, and endothelial cell transition towards a proinflammatory phenotype [40-42]. Previously, we found associations of serum MIF with pulmonary arterial hypertrophy, pulmonary vas- cular resistance, and response to inhaled nitric oxide in pediatric subjects with congenital cardiac communications [43]. Of potential interest in terms of acute changes in pulmonary vascular tone, MIF was shown to enhance pulmonary vasoconstriction in response to hypoxia and potentiate constriction preevoked by agonists in isolated pulmonary artery rings [44].

\section{Conclusion}

Based on our findings, we conclude that pulmonary hemodynamic patterns may be identified early after surgery in patients with congenital cardiac communications. A relationship was demonstrated between postoperative systemic inflammatory response and the behavior of the pulmonary circulation. Early postoperative elevation of pulmonary arterial pressure was associated with relevant cardiopulmonary events, which in turn accounted for prolonged mechanical ventilation. Six markers of inflammation present in circulation were directly or indirectly associated with the occurrence of postoperative events. The relationships that we report between hemodynamics, inflammation, and outcome may have implications in terms of treatment planning and prevention of complications in this population.

\section{Data Availability}

The data used to support the conclusion of the present study correspond to the project FAPESP \#2015/21587-5 and are available from the corresponding author upon request.

\section{Disclosure}

This work is part of a doctoral thesis by Maria F. S. Souza to the Program in Cardiology, University of São Paulo School of Medicine, São Paulo, Brazil.

\section{Conflicts of Interest}

The authors declare that they have no known competing financial interests or personal relationships that could have appeared to influence the work reported in this paper.

\section{Authors' Contributions}

Maria Francilene S. Souza performed the conceptualization, methodology, investigation, and data curation and wrote the original draft. Juliano G. Penha and Nair Y. Maeda performed the conceptualization, methodology, investigation, and data curation and wrote, reviewed, and edited the manuscript. Filomena R. B. G. Galas, Kelly C. O. Abud, and Eloisa S. Carvalho performed the methodology, investigation, and data curation and wrote, reviewed, and edited the manuscript. Ana Maria Thomaz and Claudia R. P. Castro performed the investigation and data curation and wrote, reviewed, and edited the manuscript. Juliana Pereira performed the conceptualization, methodology, investigation, and supervision and wrote, reviewed, and edited the manuscript. Antonio Augusto Lopes performed conceptualization, funding acquisition, methodology, investigation, project 
administration, supervision, and formal analysis and wrote, reviewed, and edited the manuscript.

\section{Acknowledgments}

This work was supported by FAPESP-Foundation for Research Support of the State of São Paulo (grant \# 2015/ 21587-5). We greatly acknowledge the assistance of Mrs. Roseli Polo in all phases of the study and appreciate the collaborative participation of the pediatric nurses Mrs. Janete H. Ferreira and Mrs. Luciana W. de Souza. We also acknowledge the collaborative work of pediatric cardiologists and cardiac surgeons and all colleagues of the multiprofessional team involved in assisting patients at the Heart Institute (InCor), São Paulo, Brazil.

\section{References}

[1] D. J. Kozik and J. S. Tweddell, "Characterizing the inflammatory response to cardiopulmonary bypass in children," The Annals of Thoracic Surgery, vol. 81, no. 6, pp. S2347-S2354, 2006.

[2] I. Adatia, "Pulmonary hypertension and postoperative congenital heart disease," in Pediatric Pulmonary Hypertension, M. Beghetti, Ed., pp. 209-232, Elsevier Urban \& Fischer, Munich, 2011.

[3] S. Toyama, F. Hatori, A. Shimizu, and T. Takagi, "A neutrophil elastase inhibitor, sivelestat, improved respiratory and cardiac function in pediatric cardiovascular surgery with cardiopulmonary bypass," Journal of Anesthesia, vol. 22, no. 4, pp. 341-346, 2008.

[4] Y. Durandy, "Minimizing systemic inflammation during cardiopulmonary bypass in the pediatric population," Artificial Organs, vol. 38, no. 1, pp. 11-18, 2014.

[5] M. Boehne, M. Sasse, A. Karch et al., "Systemic inflammatory response syndrome after pediatric congenital heart surgery: incidence, risk factors, and clinical outcome," Journal of Cardiac Surgery, vol. 32, no. 2, pp. 116-125, 2017.

[6] Y. Fujii, "Evaluation of inflammation caused by cardiopulmonary bypass in a small animal model," Biology, vol. 9, no. 4, p. $81,2020$.

[7] R. J. Kameny, J. Fineman, and I. Adatia, "Perioperative management of pediatric pulmonary hypertension," Advances in Pulmonary Hypertension, vol. 15, no. 2, pp. 87-91, 2016.

[8] L. Lindberg, A. K. Olsson, P. Jögi, and C. Jonmarker, "How common is severe pulmonary hypertension after pediatric cardiac surgery?," The Journal of Thoracic and Cardiovascular Surgery, vol. 123, no. 6, pp. 1155-1163, 2002.

[9] N. Brunner, V. A. de Jesus Perez, A. Richter et al., "Perioperative pharmacological management of pulmonary hypertensive crisis during congenital heart surgery," Pulmonary Circulation, vol. 4, no. 1, pp. 10-24, 2014.

[10] A. Hofer, M. Heschi, C. Kern et al., “A prospective randomised study of postoperative prophylactic application of sildenafil after pediatric cardiac surgery," Journal of Clinical \& Experimental Cardiology, vol. 6, no. 8, 2015.

[11] S. H. Abman, G. Hansmann, S. L. Archer et al., "Pediatric Pulmonary Hypertension: Guidelines From the American Heart Association and American Thoracic Society," Circulation, vol. 132, no. 21, pp. 2037-2099, 2015.
[12] D. Kolte, S. Lakshmanan, M. D. Jankowich, E. L. Brittain, B. A. Maron, and G. Choudhary, "Mild pulmonary hypertension is associated with increased mortality: a systematic review and meta-analysis," Journal of the American Heart Association, vol. 7, no. 18, article e009729, 2018.

[13] M. G. Gaies, H. E. Jeffries, R. A. Niebler et al., "Vasoactive-inotropic score is associated with outcome after infant cardiac surgery: an analysis from the Pediatric Cardiac Critical Care Consortium and Virtual PICU System Registries," Pediatric Critical Care Medicine, vol. 15, no. 6, pp. 529-537, 2014.

[14] Z. V. Ribeiro and J. M. Tsutsui, "Doppler echocardiography and hemodynamic parameters in congenital heart disease with increased pulmonary flow," Arquivos Brasileiros de Cardiologia, vol. 94, no. 5, pp. 592-600, 2010.

[15] I. R. Rivera, M. A. Mendonça, J. L. Andrade et al., "Pulmonary venous flow index as a predictor of pulmonary vascular resistance variability in congenital heart disease with increased pulmonary flow: a comparative study before and after oxygen inhalation," Echocardiography, vol. 30, no. 8, pp. 952-960, 2013.

[16] M. Koestenberger, W. Ravekes, A. D. Everett et al., "Right ventricular function in infants, children and adolescents: reference values of the tricuspid annular plane systolic excursion (TAPSE) in 640 healthy patients and calculation of Z-score values," Journal of the American Society of Echocardiography, vol. 22, no. 6, pp. 715-719, 2009.

[17] D. L. Burke, M. G. Frid, C. L. Kunrath et al., "Sustained hypoxia promotes the development of a pulmonary arteryspecific chronic inflammatory microenvironment," American Journal of Physiology. Lung Cellular and Molecular Physiology, vol. 297, no. 2, pp. L238-L250, 2009.

[18] W. L. Chandler and T. Velan, "Secretion of tissue plasminogen activator and plasminogen activator inhibitor 1 during cardiopulmonary bypass," Thrombosis Research, vol. 112, no. 3, pp. 185-192, 2003.

[19] R. Tu, Y. Peng, Y. Wang, X. Tang, and S. Wang, “The stromal cell-derived factor $1 / \mathrm{C}-\mathrm{X}-\mathrm{C}$ chemokine receptor type 4 axis is important in neutrophil migration caused by cardiopulmonary bypass in children," Interactive Cardiovascular and Thoracic Surgery, vol. 26, no. 3, pp. 431-437, 2018.

[20] D. Lievens, A. Zernecke, T. Seijkens et al., "Platelet CD40L mediates thrombotic and inflammatory processes in atherosclerosis," Blood, vol. 116, no. 20, pp. 4317-4327, 2010.

[21] K. Büchner, V. Henn, M. Gräfe, O. J. de Boer, A. E. Becker, and R. A. Kroczek, "CD40 ligand is selectively expressed on CD4+ T cells and platelets: implications for CD40-CD40L signalling in atherosclerosis," The Journal of Pathology, vol. 201, no. 2, pp. 288-295, 2003.

[22] M. F. S. Souza, E. S. Carvalho, N. Y. Maeda et al., "Macrophage migration inhibitory factor and chemokine RANTES in young pediatric patients with congenital cardiac communications: relation to hemodynamic parameters and the presence of Down syndrome," Cytokine, vol. 134, article 155192, 2020.

[23] M. Nakano, T. Fujii, M. Hashimoto et al., "Type I interferon induces CX3CL1 (fractalkine) and CCL5 (RANTES) production in human pulmonary vascular endothelial cells," Clinical and Experimental Immunology, vol. 170, no. 1, pp. 94-100, 2012.

[24] C. Bakogiannis, M. Sachse, K. Stamatelopoulos, and K. Stellos, "Platelet-derived chemokines in inflammation and atherosclerosis," Cytokine, vol. 122, article 154157, 2019. 
[25] D. Lotan, D. Zilberman, O. Dagan et al., “ $\beta$-Chemokine secretion patterns in relation to clinical course and outcome in children after cardiopulmonary bypass: continuing the search to abrogate systemic inflammatory response," The Annals of Thoracic Surgery, vol. 71, no. 1, pp. 233-237, 2001.

[26] H. Ide, T. Kakiuchi, N. Furuta et al., "The effect of cardiopulmonary bypass on $\mathrm{T}$ cells and their subpopulations," The Annals of Thoracic Surgery, vol. 44, no. 3, pp. 277-282, 1987.

[27] R. Natanov, F. Gueler, C. S. Falk et al., "Blood cytokine expression correlates with early multi-organ damage in a mouse model of moderate hypothermia with circulatory arrest using cardiopulmonary bypass," PLoS One, vol. 13, no. 10, article e0205437, 2018.

[28] E. M. Boneberg and T. Hartung, "Molecular aspects of antiinflammatory action of G-CSF," Inflammation Research, vol. 51, no. 3, pp. 119-128, 2002.

[29] A. Parmigiani, M. Lichtenheld, and S. Pahwa, "IL-21 reduces production of pro-inflammatory cytokines by activated CD8 T cells," Journal of Immunology, vol. 188, 1 Supplement, 2012.

[30] A. Korniluk, O. M. Koper-Lenkiewicz, J. Kamińska, H. Kemona, and V. Dymicka-Piekarska, "Mean platelet volume (MPV): new perspectives for an old marker in the course and prognosis of inflammatory conditions," Mediators of Inflammation, vol. 2019, Article ID 9213074, 14 pages, 2019.

[31] W. T. McBride, M. A. Armstrong, H. Gilliland, and T. J. McMurray, "The balance of pro and anti-inflammatory cytokines in plasma and bronchoalveolar lavage (BAL) at paediatric cardiac surgery," Cytokine, vol. 8, no. 9, pp. 724-729, 1996.

[32] T. Kawamura, R. Wakusawa, and K. Inada, "Interleukin-10 and interleukin-1 receptor antagonists increase during cardiac surgery," Canadian Journal of Anaesthesia, vol. 44, no. 1, pp. 38-42, 1997.

[33] W. P. Arend, "The balance between IL-1 and IL-1Ra in disease," Cytokine \& Growth Factor Reviews, vol. 13, no. 4-5, pp. 323-340, 2002.

[34] G. Justus, C. Walker, L. M. Rosenthal, F. Berger, O. Miera, and K. R. L. Schmitt, "Immunodepression after CPB: cytokine dynamics and clinics after pediatric cardiac surgery - a prospective trial," Cytokine, vol. 122, article 154018, 2019.

[35] N. F. Voelkel, R. M. Tuder, J. Bridges, and W. P. Arend, "Interleukin-1 receptor antagonist treatment reduces pulmonary hypertension generated in rats by monocrotaline," American Journal of Respiratory Cell and Molecular Biology, vol. 11, no. 6, pp. 664-675, 1994.

[36] M. Chada, S. Nögel, A. M. Schmidt et al., "Anakinra (IL-1R antagonist) lowers pulmonary artery pressure in a neonatal surfactant depleted piglet model," Pediatric Pulmonology, vol. 43, no. 9, pp. 851-857, 2008.

[37] C. B. Bui, M. Kolodziej, E. Lamanna et al., "Interleukin-1 receptor antagonist protects newborn mice against pulmonary hypertension," Frontiers in Immunology, vol. 10, p. 1480, 2019.

[38] T. Calandra and T. Roger, "Macrophage migration inhibitory factor: a regulator of innate immunity," Nature Reviews. Immunology, vol. 3, no. 10, pp. 791-800, 2003.

[39] N. C. Riedemann, R. F. Guo, and P. A. Ward, "Novel strategies for the treatment of sepsis," Nature Medicine, vol. 9, no. 5, pp. 517-524, 2003.

[40] B. Zhang, M. Shen, M. Xu et al., "Role of macrophage migration inhibitory factor in the proliferation of smooth muscle cell in pulmonary hypertension," Mediators of Inflammation, vol. 2012, Article ID 840737, 10 pages, 2012.
[41] L. Tu, A. Huertas, E. Fadel et al., "CD74-dependent interleukin- 6 and monocyte chemoattractant protein-1 secretion by pulmonary endothelial cells in idiopathic pulmonary hypertension," American Journal of Respiratory and Critical Care Medicine, vol. 187, p. A1229, 2012.

[42] M. Le Hiress, L. Tu, N. Ricard et al., "Proinflammatory signature of the dysfunctional endothelium in pulmonary hypertension. Role of the macrophage migration inhibitory factor/ CD74 complex," American Journal of Respiratory and Critical Care Medicine, vol. 192, no. 8, pp. 983-997, 2015.

[43] N. Y. Maeda, V. D. Aiello, P. C. Santos et al., "Relation of macrophage migration inhibitory factor to pulmonary hemodynamics and vascular structure and carbamyl-phosphate synthetase I genetic variations in pediatric patients with congenital cardiac shunts," Mediators of Inflammation, vol. 2019, Article ID 7305028, 10 pages, 2019.

[44] B. Zhang, Y. Luo, M. L. Liu et al., "Macrophage migration inhibitory factor contributes to hypoxic pulmonary vasoconstriction in rats," Microvascular Research, vol. 83, no. 2, pp. 205-212, 2012. 